\title{
Global stability criterion for a quantum feedback control process on a single qubit and exponential stability in case of perfect detection efficiency
}

\author{
Andreas de Vries* \\ FH Südwestfalen University of Applied Sciences, \\ Haldener Straße 182, D-58095 Hagen, Germany
}

(Dated: September 1, 2018)

\begin{abstract}
Quantum feedback control is a technology which can be used to drive a quantum system into a predetermined eigenstate. In this article, sufficient conditions for the experiment parameters of a quantum feedback control process of a homodyne QND measurement are given to guarantee feedback control of a spin-1/2 quantum system in case of imperfect detection efficiency. For the case of pure states and perfect detection efficiency, time scales of feedback control processes are calculated.

PACS numbers: 03.65.Ta, 42.50.Lc, 02.30.Yy
\end{abstract}

\section{INTRODUCTION}

In classical control theory, feedback control describes processes in which a closed-loop controller is used to steer the states or outputs of a dynamical system, which in turn effect the inputs of the controller into the system. A remarkable approach to feedback control of quantum spin systems has recently been elaborated in [1]. Here QND measurements are utilized to let a quantum system collapse deterministically onto a predetermined eigenstate.

In the present article, the stability and the time scale of quantum feedback control processes are studied. As a result (Theorem 2), sufficient limits for the experiment control parameters are derived to guarantee asymptotically stable quantum feedback control processes on a spin- $\frac{1}{2}$ quantum system. It is proved by applying Lyapunov's method to the stochastic differential equation governing the quantum state evolution, and thus differs from the similar result in [1] proposing numerical methods of semialgebraic geometry and aiming at applicability for higher spin systems where efficient search for Lyapunov functions is practically impossible. For the special case of pure states and perfect detection efficiency, the quantum feedback control process is proved to terminate even exponentially fast in time.

The article is organized as follows. First, the notions of QND measurements and quantum feedback control for a spin- $\frac{1}{2}$ system are shortly reviewed, before the stochastic stability of quantum feedback control processes with imperfect and perfect detection efficiency are studied, and the results are shortly discussed.

\section{QND MEASUREMENTS}

In contrast to a measurement in classical physics, a quantum measurement inevitably changes, or even de-

\footnotetext{
*Electronic address: de-vries@fh-swf.de

${ }^{\dagger}$ Also at AG Mathem. Physik, Ruhr-Universität, D-44780 Bochum
}

stroys, the measured quantum system itself [2, [3]. Theoretical as well as experimental investigation have been intensively made dealing with processes where quantum measurements are utilized constructively, for instance theoretical considerations of measurement determination by the quantum register [4], quantum feedback control by continuous measurements $[5,[6,7,6]$, especially in quantum optics 1, 9, 10, 11, stabilization and purification of two-level systems [12, 13], conditional measurements of coupled quantum dots by a point contact detector [14, 15] or by a SET [16, 17, 18], and the conditional measurement approach due to Sherman and Kurizki 19] to prepare predetermined field states of atoms trapped in optical QED cavities [20, 21, 22], as well as a similar approach analyzed for spin squeezing in Cs clocks [23].

Although these approaches differ considerably in detail, most of them utilize repeated quantum nondemolition (QND) measurements [24, §3.3], i.e., measurements of an observable $Y$ satisfying the self-nondemolition condition $\left[Y(t), Y\left(t^{\prime}\right)\right]=0$ for all times $t, t^{\prime}$, as well as the back action evasion condition $\left[Y, H_{\mathrm{int}}\right]=0$, where $H_{\text {int }}=\sum_{j}|j\rangle\langle j| \otimes B_{j}$ denotes the interaction Hamiltonian between the considered system (the projections $|j\rangle\langle j|)$ and the measuring apparatus $\left(B_{j}\right)$. The QND observable $Y$ may correspond, for instance, to a Hermitian Lindblad operator $L$, or to a conserved quantity, such as a constant of motion of the considered system like polarization or momentum.

\section{QUANTUM FEEDBACK CONTROL}

Due to ideas of Belavkin [5, [6, 7] as well as Wiseman and coworkers [8, 25], an approach to quantum feedback control of spin systems has been recently developed by van Handel, Stockton, and Mabuchi [1]. In this approach repeated quantum nondemolition measurements are engineered to let quantum spin systems collapse deterministically onto a previously chosen eigenstate. This idea, surprising from a traditional physics perspective, bases on the fact that realistic measurements are not instantaneous but take some finite time. If these reduction time 
scales are of an order attainable by modern digital electronics, a quantum filter and a controller can respond on the spin system state, feeding back the intermediate nondemolition measurement results to a Hamiltonian parameter. In this way it is possible, for instance, to deterministically prepare highly entangled Dicke states [11], to generate and utilize squeezed quantum states of trapped atoms in an optical cavity [23], or to improve quantum error correction [26].

The quantum stochastic control formalism of van Handel, Stockton, and Mabuchi [1] can be considered as an extension of probability theory, and the traditional formulation of quantum mechanics can be directly recovered from it. In [1, §IV.C] a stabilizing controller is given for a quantum system of $\operatorname{spin} j=\frac{1}{2}$, schematically depicted in Figure 1. The conditional evolution of the density op-

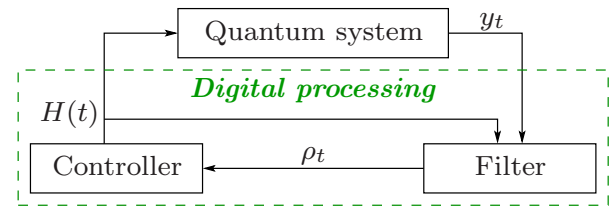

FIG. 1: (Color online) Schema of a quantum feedback control process. The QND measurement output $y_{t}$ from the quantum system is used to propagate the conditional state of the filter, via the feedback signal $H(t)$. The dashed line indicates (classical) digital processing, the filter is determined by Eq. (11).

erator $\rho$ describing the quantum system depends on the probe parameter measurement rate $M>0$ in $\mathrm{Hz}$, and the detection efficiency $\eta \in[0,1]$, a pure number. More precisely, the conditional evolution of $\rho$ is determined by the stochastic master equation

$$
\mathrm{d} \rho_{t}=\mathscr{G}^{*}[H(t), L] \rho_{t} \mathrm{~d} t+\sqrt{\eta} \mathscr{H}[L] \rho_{t} \mathrm{~d} W_{t},
$$

where $H(t)$ is the control Hamiltonian (with $H(t)=0$ in case of no feedback), $L$ is an observable one of whose eigenstates is the desired final state of the system, $\mathscr{G}^{*}=$ $\mathscr{G}^{*}[H(t), L]$ is the adjoint generator

$$
\mathscr{G}^{*} \rho_{t}=-\mathrm{i}\left[H(t), \rho_{t}\right]+L \rho_{t} L^{*}-\frac{1}{2}\left(L^{*} L \rho_{t}+\rho_{t} L^{*} L\right),
$$

$\mathscr{H}$ is the superoperator

$$
\mathscr{H}[L] \rho_{t}=L \rho_{t}+\rho_{t} L^{*}-\operatorname{Tr}\left[\rho_{t}\left(L+L^{*}\right)\right] \rho_{t},
$$

and $\mathrm{d} W_{t}$ is the innovations process

$$
\mathrm{d} W_{t}=2 \sqrt{M \eta} y_{t} \mathrm{~d} t-\sqrt{\eta} \operatorname{Tr}\left[\rho_{t}\left(L+L^{*}\right)\right] \mathrm{d} t
$$

depending on the QND measurement record $y_{t}$ of the output corresponding to the observable $Y(t)[11, \S I I]$. Here $Y$ is normalized (i.e., $\mathrm{d} Y_{t}^{2}=\mathrm{d} t$ ) and related to $L$ and the standard noises $A$ and $A^{*}$ by the Hudson-Parthasarathy equation. The innovations $\mathrm{d} W_{t}$ is a Wiener increment and $\mathrm{d} W_{t} / \mathrm{d} t$ is a Gaussian white noise. Note that $\mathrm{d} W_{t}$ is one-dimensional, whereas Eq. (1) is operator-valued.

Usually, the controller Hamiltonian $H(t)$ is determined by a few control parameters. It is most desirable if they could be adjusted in a way such that the master equation has an asymptotically stable fixed point.

\section{A. Density operator space of a spin- $\frac{1}{2}$ system}

In the prototypical physical model of homodyne measurement of a spin system [1], where $y_{t}$ denotes the homodyne measurement record of the output, the observable $L$ and the controller Hamiltonian $H(t)$ in Eq. (10) are given by

$$
L=\sqrt{M} J_{z} \quad \text { and } \quad H(t)=B(t) J_{y}
$$

with the usual angular momentum observables $J_{y}, J_{z}$, and $\mathrm{d} W_{t} / \mathrm{d} t$ can be identified with the shot-noise of the homodyne local oscillator. Here $M>0$ is the strength of the interaction between the light and the atoms and is regulated experimentally by the optical cavity, and the control input $B(t)$ is the applied magnetic field. The time scale then only depends on the sensitivity $1 /(2 \sqrt{M \eta})$ of the photodetection per $\sqrt{\mathrm{Hz}}$, and the feedback gain parameters of the controller $B(t)$. Hence the time scale only depends on experimentally controlled parameters. For perfect detection efficiency, $\eta=1$, the stochastic master equation governing a pure quantum system under feedback control is one-dimensional and will be tackled analytically below.

For the special case of a quantum system of spin $\frac{1}{2}$, i.e., a qubit, the space of all density operators $\rho$ of the system is represented by the two-dimensional disc

$$
D^{2}=\left\{(\lambda, \nu) \in \mathbb{R}^{2}: \lambda^{2}+\nu(\nu-1) \leqq 0\right\}
$$

with center $\left(0, \frac{1}{2}\right)$ and radius $\frac{1}{2},[1, \S I V . C]$. Here the density matrix entries are given by $\rho_{11}=\nu, \rho_{22}=1-\nu$, $\rho_{21}=\rho_{12}^{*}=\lambda$, and the state $(\lambda, \nu)=(0,0)$ to be stabilized corresponds to $\rho=|1\rangle\langle 1|=\operatorname{diag}(0,1)$. Note that

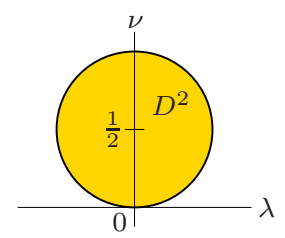

FIG. 2: (Color online) The density operator space $D^{2}$ of a spin- $\frac{1}{2}$ quantum system. The origin $(\lambda, \nu)=(0,0)$ corresponds to the quantum state $|1\rangle\langle 1|$, the point $(0,1)$ to $|0\rangle\langle 0|$.

in this case the imaginary parts of the off-diagonal entries of $\rho$ decouple and may be neglected. The stochastic master equation (11) describing the conditional evolution of a single qubit thus is reducible to the two-dimensional Itô equation [1]

$$
\begin{aligned}
\mathrm{d} \lambda_{t} & =\left[B(t)\left(\nu_{t}-\frac{1}{2}\right)-\frac{M}{2} \lambda_{t}\right] \mathrm{d} t+\sqrt{M \eta} \lambda_{t}\left(1-2 \nu_{t}\right) \mathrm{d} W_{t}, \\
\mathrm{~d} \nu_{t} & =-B(t) \lambda_{t} \mathrm{~d} t-2 \sqrt{M \eta} \nu_{t}\left(\nu_{t}-1\right) \mathrm{d} W_{t} .
\end{aligned}
$$

Its infinitesimal generator 27, $\$ 7.3]$ is given as

$$
\begin{gathered}
\mathscr{L}=\left[B(\lambda, \nu)\left(\nu-\frac{1}{2}\right)-\frac{M}{2} \lambda\right] \frac{\partial}{\partial \lambda}-B(\lambda, \nu) \lambda \frac{\partial}{\partial \nu} \\
+2 M \eta\left[\lambda^{2}\left(\nu-\frac{1}{2}\right)^{2} \frac{\partial^{2}}{\partial \lambda^{2}}+\nu^{2}(\nu-1)^{2} \frac{\partial^{2}}{\partial \nu^{2}}\right], \\
\text { so } \frac{\mathrm{d} \mathbb{E}\left[f\left(x_{t}\right)\right]}{\mathrm{d} t}=\mathbb{E}\left[\mathscr{L} f\left(x_{t}\right)\right] .
\end{gathered}
$$




\section{STOCHASTIC STABILITY OF QUANTUM FEEDBACK CONTROL PROCESSES}

\section{A. Stochastic stability}

In control theory stabilization of nonlinear systems is usually investigated using Lyapunov theory. In the 1960s, the stochastic counterpart of Lyapunov theory [28] was developed by Has'minskiř and others. To prove the central results of this article, we first have to define asymptotic stability of stochastic processes.

Definition 1 Let $W_{t}$ be a Wiener process on the canonical Wiener space $(\Omega, \mathscr{F}, \mathbb{P})$, and let $x$ obey the Itô equation on $\mathbb{R}^{n}$,

$$
\mathrm{d} x_{t}=b\left(x_{t}\right) \mathrm{d} t+\sigma\left(x_{t}\right) \mathrm{d} W_{t},
$$

where $b, \sigma: \mathbb{R}^{n} \rightarrow \mathbb{R}^{n}$ satisfy the usual growth and Lipschitz conditions for existence and uniqueness of solutions [27]. Then an equlibrium solution $x_{*}$ of Eq. (9), i.e., a solution satisfying $b\left(x_{*}\right)=\sigma\left(x_{*}\right)=0$, is called stable in probability if

$$
\lim _{x_{0} \rightarrow x_{*}} \mathbb{P}\left[\sup _{t \geqq 0}\left|x_{t}-x_{*}\right|>\varepsilon\right]=0 \quad \forall \varepsilon>0 .
$$

It is called asymptotically stable if it is stable in probability and

$$
\lim _{x_{0} \rightarrow x_{*}} \mathbb{P}\left[\lim _{t \rightarrow \infty}\left|x_{t}-x_{*}\right|=0\right]=1 .
$$

It is called globally stable if it is stable in probability and

$$
\mathbb{P}\left[\lim _{t \rightarrow \infty}\left|x_{t}-x_{*}\right|=0\right]=1 \text {. }
$$

$x_{*}$ is called exponentially stable in $p$-th moment, $p \in \mathbb{N}$, [29] if there exists a pair of constants $a, \alpha>0$ such that

$$
\mathbb{E}\left[\left|x_{t}-x_{*}\right|^{p}\right] \leqq a \mathbb{E}\left[\left|x_{0}-x_{*}\right|^{p}\right] \mathrm{e}^{-\alpha t}
$$

for all $t \geqq 0$. Especially for $p=1, x_{*}$ is then called exponentially stable in mean, and for $p=2$ exponentially stable in mean square. The smallest possible value of the constant $a$ is referred to as the growth constant, and the largest possible value of $\alpha$ as the rate constant or rate of convergence.

The first two notions are local properties, whereas the third one is a global property of the system.

\section{B. Imperfect detection efficiency}

If quantum feedback control is performed with only imperfect detection efficiency, i.e., $0<\eta<1$, the following theorem yields a sufficient condition for the global asymptotical stability of its final state. Although a similar result for a special controller has been shown already in [1, $\S$ IV.F] by numerical semialgebraic methods, here a general analytical criterion relating the controller parameters is given.
Theorem 2 Consider a quantum feedback control process of a spin- $\frac{1}{2}$ quantum system, described by the density operators in the state space $D^{2}$ and by the stochastic master equation (7), with the probe parameter measurement rate $M>0$, the detection efficiency $\eta \in(0,1]$ and the controller

$$
B(\lambda, \nu)=g_{1} \lambda+g_{2} \nu
$$

Assume that for the feedback gain parameters $g_{1}$ and $g_{2}$ there exist real constants $c>1,0<d<2(c-1)$ such that the maximum $f_{\max }$ of the auxiliary function $f:\left[0, \frac{1}{2}\right] \times$ $[-\pi, \pi] \rightarrow \mathbb{R}$

$$
\begin{aligned}
f(r, \theta)= & {\left[M-(c-1) g_{1}\right](1-\cos \theta)-d g_{1} r \sin \theta(1-\cos \theta) } \\
& +\left[d g_{1}(1+\cos \theta) r-(c-1) g_{2}-\frac{d\left(g_{1}+M\right)}{2}\right] \sin \theta \\
& +2 d g_{2}(1+\cos \theta)\left(2 r \cos \theta-\frac{1}{2}\right) \\
& -4 M \eta(1-\cos \theta)\left((1+\cos \theta) r-\frac{1}{2}\right)^{2} \\
& -4 M \eta(1+\cos \theta)((1+\cos \theta) r-1)^{2},
\end{aligned}
$$

is negative, i.e., $f_{\max }<0$. Then the state $(0,0) \in D^{2}$, corresponding to $\rho_{0}=|1\rangle\langle 1|$, of the quantum system undergoing a quantum feedback control process is globally stable. Moreover, condition (15) implies

$$
g_{1}>\frac{(1-\eta) M}{c-1} \geqq 0, \quad-\frac{4 M \eta}{d}<g_{2}<0 .
$$

Proof. Defining the function

$$
V(\lambda, \nu)=c \nu+d \lambda \nu-\lambda^{2}-\nu^{2},
$$

we have $V(0,0)=0$ and $V(\lambda, \nu)>0$ for $(\lambda, \nu) \in D^{2} \backslash\{\mathbf{0}\}$. Since $0 \leqq \lambda^{2} \leqq \nu(1-\nu)$ and $\lambda \geqq-\frac{1}{2}$, we have $V=$ $(c-1) \nu+d \lambda \nu-\left(\lambda^{2}+\nu(\nu-1)\right) \geqq(c-1) \nu-\frac{d}{2} \nu$, i.e.,

$$
V(\lambda, \nu) \geqq\left(c-\frac{d}{2}-1\right) \nu,
$$

and especially $V>0$ for $\nu>0$. Moreover by (14),

$$
\begin{aligned}
\mathscr{L} V= & \left(g_{1} \lambda+g_{2} \nu\right)\left[\left(d\left(\nu^{2}-\frac{\nu}{2}-\lambda^{2}\right)-(c-1) \lambda\right]\right. \\
& -\frac{M}{2} \lambda(d \nu-2 \lambda) \\
& -4 M \eta\left[\lambda^{2}\left(\nu-\frac{1}{2}\right)^{2}+\nu^{2}(\nu-1)^{2}\right] \\
= & \left.M-d\left(g_{1} \lambda+g_{2} \nu\right)-(c-1) g_{1}\right] \lambda^{2}+d g_{2} \nu^{2}\left(\nu-\frac{1}{2}\right) \\
& +\left[d g_{1}\left(\nu-\frac{1}{2}\right)-(c-1) g_{2}-\frac{d M}{2}\right] \lambda \nu \\
& -4 M \eta\left[\lambda^{2}\left(\nu-\frac{1}{2}\right)^{2}+\nu^{2}(\nu-1)^{2}\right] .
\end{aligned}
$$

We see immediately that $\mathscr{L} V(0,0)=0$. We will prove next that $\mathscr{L} V(\lambda, \nu)<0$ for $(\lambda, \nu) \in D^{2} \backslash\{\mathbf{0}\}$. Using the coordinates $(r, \theta)$, where $r \in\left(0, \frac{1}{2}\right]$ and $\theta \in(-\pi, \pi)$, given by $r=\frac{\lambda^{2}+\nu^{2}}{2 \nu}, \tan \frac{\theta}{2}=\frac{\lambda}{\nu}$, we have $(\lambda, \nu)=r(\sin \theta, 1+$ $\cos \theta)$. Thus we obtain

$$
f(r, \theta)=\frac{\mathscr{L} V(\lambda(r, \theta), \nu(r, \theta))}{r^{2}(1+\cos \theta)} .
$$

By the assumption of the Theorem, $f(r, \theta)<0$ for $(r, \theta) \in\left[0, \frac{1}{2}\right] \times(-\pi, \pi)$, hence $\mathscr{L} V(\lambda, \nu)<0$ on $D^{2} \backslash\{\mathbf{0}\}$. 
Therefore, $V$ is a strict Lyapunov function on $D^{2}$ with the only asymptotically stable state $(\lambda, \nu)=0$.

Since $f(r, \pm \pi)=2\left((1-\eta) M-(c-1) g_{1}\right)$, as well as $f(r, 0)=-32 M \eta\left(r^{2}-\left(1+\frac{d g_{2}}{4 M \eta}\right) r+\frac{4 M \eta+d g_{2}}{16 M \eta}\right)$, assumption (15) implies (16).

Therefore, a quantum feedback control process satisfying the assumptions of Theorem 2 drives a spin- $\frac{1}{2}$ quantum system to the state $\left(\begin{array}{l}0 \\ 0\end{array}\right) \in D^{2}$, no matter in which quantum state the system is initially. This holds true even for the worst case, when the initial state is $\rho_{i}=|0\rangle\langle 0|=\left(\begin{array}{l}0 \\ 1\end{array}\right) \in D^{2}$. Note that a standard state reduction measurement would leave the quantum system in this state with certainty.

The next example shows that there indeed exist parameter constellations satisfying Theorem 2 .

Example 3 For the experimentally controlled parameters $g_{1}=\frac{3 M}{4}, g_{2}=-\frac{M}{4}$, and $\eta=\frac{1}{2}$, and the constants
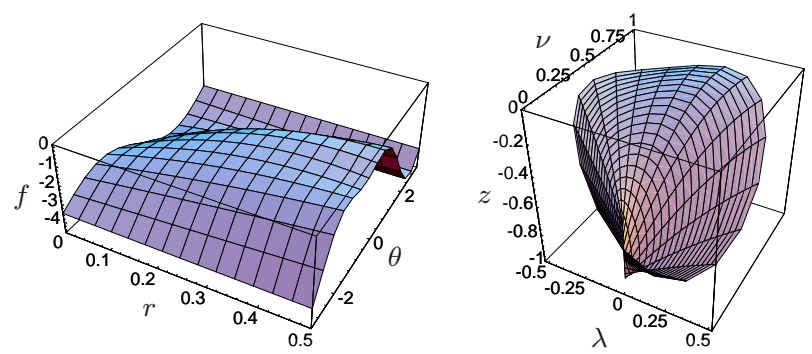

FIG. 3: (Color online) The auxiliary function $h$ in (15) for $g_{1}=\frac{3 M}{4}, g_{2}=-\frac{M}{4}, \eta=\frac{M}{2}, c=4, d=2$. Left figure: The graph of $f(r, \theta)$. Right figure: The corresponding 2-manifold imbedded in 3 -space $(\lambda, \nu, z)$, parametrized by $\lambda=r \sin \theta$, $\nu=(1+\cos \theta) r, z=\frac{f(r, \theta)}{5}$ for $0<r \leqq \frac{1}{2},|\theta|<\pi$.

$c=4, d=2$, the function $h$ in (15) is negative on $D^{2} \backslash\{\mathbf{0}\}$, which may be seen graphically (Fig. 3).

\section{Pure states and perfect detection efficiency}

For pure quantum states and perfect detection efficiency, we are able to estimate the expected running time for a quantum feedback control process more precisely, as is shown in the following theorem.

Theorem 4 Consider a quantum feedback control process of a spin- $\frac{1}{2}$ quantum system of pure quantum states, described by the stochastic master equation (7) with the probe parameter measurement rate $M>0$, the perfect detection efficiency $\eta=1$, and the controller $B(t)$ of 14 with feedback gain parameters

$$
g_{1}>0, \quad g_{2}<0 .
$$

Then this process has an expected running time

$$
T_{\mathrm{qfc}}\left(M, g_{1}\right)=O\left(\mathrm{e}^{-\left(g_{1}+M\right) t / 2}\right),
$$

where $t$ denotes the time duration since the process start. In particular, $\mathbf{0} \in D^{2}$ is globally exponentially stable in mean.

Proof. For a quantum feedback control process of a spin$\frac{1}{2}$ system, performed with perfect efficiency, the space of all density operators $\rho$ of pure states of the system can be reduced to the circle

$$
S^{1}=\left\{(\sin \theta, 1+\cos \theta) \in \mathbb{R}^{2}: \theta \in(-\pi, \pi]\right\}
$$

with center $\left(0, \frac{1}{2}\right)$ and radius $\frac{1}{2},[1, \S I V . C]$, and the stochastic master equation (1) describing the conditional evolution of a single qubit, with perfect efficiency $\eta=1$, then is reducible to the one-dimensional Itô equation

$$
\mathrm{d} \theta_{t}=\left(B(t)-\frac{M}{2} \sin \theta_{t} \cos \theta_{t}\right) \mathrm{d} t-\sqrt{M} \sin \theta_{t} \mathrm{~d} W_{t} .
$$

Choosing the controller as

$$
B(t)=\frac{g_{1}}{2} \sin \theta_{t}+\frac{g_{2}}{2}\left(1+\cos \theta_{t}\right),
$$

the system stabilizes the state $\theta= \pm \pi$, which we mark as $|1\rangle\langle 1|$. Eq. (24) has a unique solution on the interval $[-\pi, \pi]$ because its coefficients satisfy the sufficient Lipschitz and growth conditions [27, Theor. 5.2.1]. Since the diffusion coefficient

$$
\sigma(\theta)=\sqrt{M} \sin \theta
$$

vanishes at $\theta=0$, we have to consider the two intervals $J^{-}=(-\pi, 0)$ and $J^{+}=(0, \pi)$ separately. Since moreover the drift coefficient

$$
b(\theta)=B(\theta)-\frac{M}{2} \sin \theta \cos \theta
$$

satisfies $b(-\pi)=b(\pi)=0$ and $b(0)=g_{2}<0$, the state $\theta=0$ is a reflecting barrier ('entrance boundary') for states in $J^{-}$, but an absorbing barrier ('exit boundary') for states in $J^{+}$, whereas $\theta= \pm \pi$ both are absorbing barriers [30, §5.2.1].

To estimate the expected time that a given pure state requires to reach the desired state $|1\rangle\langle 1|$, represented by $\theta= \pm \pi$, we have to compute the expected first exit time $T$ for the random variable $\theta$ to leave the interval $J^{-}$or $J^{+}$, respectively. $T$ is the solution of the inhomogeneous linear differential equation [30, §5.2.7], [31, §10.9]

$$
\frac{\partial T}{\partial t}+\frac{1}{2} \sigma^{2}(\theta) \frac{\partial^{2} T}{\partial \theta^{2}}+b(\theta) \frac{\partial T}{\partial \theta}=-1
$$

on $J^{ \pm}$under the boundary conditions

$$
T( \pm \pi, t)=T(0+, t)=\frac{\partial}{\partial \theta} T(0-, t)=0
$$

and $T(\theta, 0)=f(\theta)$. With the change of variable

$$
x=\frac{1+\cos \theta}{\sin \theta}=\cot \frac{\theta}{2}
$$


we obtain the relations $\sin \theta=\frac{2 x}{1+x^{2}}$ and $\cos \theta=\frac{x^{2}-1}{x^{2}+1}$, i.e., $\frac{\sigma^{2}}{2}=\frac{2 M x^{2}}{\left(1+x^{2}\right)^{2}}$ and $b=\frac{2 g_{1} x}{1+x^{2}}+\frac{2 g_{2} x^{2}}{1+x^{2}}-\frac{M\left(x-x^{2}\right)}{\left(1+x^{2}\right)^{2}}$. Moreover, $\mathrm{d} \theta=-\frac{2 \mathrm{~d} x}{1+x^{2}}$, i.e.,

$$
\frac{\partial}{\partial \theta}=-\frac{1+x^{2}}{2} \frac{\partial}{\partial x}, \quad \frac{\partial^{2}}{\partial \theta^{2}}=\frac{\left(1+x^{2}\right)^{2}}{4} \frac{\partial^{2}}{\partial x^{2}}-\frac{x\left(1+x^{2}\right)}{2} \frac{\partial}{\partial x} .
$$

Hence Eq. (28) is rewritten as $\frac{\partial T}{\partial t}-\frac{M}{2} L T=-1$, where

$$
L=-x^{2} \frac{\partial^{2}}{\partial x^{2}}+h(x) \frac{\partial}{\partial x}
$$

with

$$
h(x)=\frac{g_{1}}{M} x+\frac{g_{2}}{M} x^{2}+\frac{x-3 x^{3}}{1+x^{2}},
$$

and where the boundary conditions $T(0, t)=0$, $T(x, t) \rightarrow 0$ as $x \rightarrow \infty$, and $\frac{\partial}{\partial x} T(x, t) \rightarrow 0$ as $x \rightarrow-\infty$ hold. Since for $|x|<1$ we have $\frac{1}{1+x^{2}}=\sum_{0}^{\infty}(-1)^{\nu} x^{2 \nu}$, we can write $h$ as

$$
h(x)=\frac{g_{1}}{M} x+\frac{g_{2}}{M} x^{2}+\left(x-3 x^{3}\right) \sum_{\nu=0}^{\infty}(-1)^{\nu} x^{2 \nu}
$$

for $|x|<1$. An eigenvalue $\lambda$ of $L$ is given by the equation $L y=\lambda y$. We use the ansatz $y(x)=\sum_{0}^{\infty} a_{k} x^{k}$. By the boundary conditions, $y(0)=0$, hence $a_{0}=0$. Moreover, $y^{\prime}(x)=\sum_{1}^{\infty} k a_{k} x^{k-1}$, and $y^{\prime \prime}(x)=\sum_{2}^{\infty} k(k-1) a_{k} x^{k-2}$, hence

$$
L y(x)=\sum_{k=2}^{\infty} k(k-1) a_{k} x^{k}+h(x) \sum_{k=1}^{\infty} k a_{k} x^{k-1} .
$$

For $|x|<1$, the series $\sum_{0}^{\infty}(-1)^{\nu} x^{2 \nu}$ is absolutely convergent and we have $\left(\sum_{1}^{N} k a_{k} x^{k-1}\right)\left(\sum_{0}^{\infty}(-1)^{\nu} x^{2 \nu}\right)=$ $\sum_{1}^{N} c_{k}$ with $c_{k}=k a_{k} \sum_{\nu=0}^{k}(-1)^{k-\nu} x^{3 k-2 \nu-1}$, i.e., $c_{1}=$ $a_{1}\left(1-x^{2}\right), c_{2}=2 a_{2}\left(x-x^{3}+x^{5}\right), c_{3}=3 a_{3}\left(x^{2}-x^{4}+\right.$ $\left.x^{6}-x^{8}\right)$. Hence $\operatorname{Ly}(x)$ has the following coefficients for the first powers of $x$,

$$
\begin{aligned}
x: & \left(\frac{g_{1}}{M}+1\right) a_{1} \\
x^{2}: & 2\left(\frac{g_{1}}{M}+2\right) a_{2}+\frac{g_{2}}{M} a_{1} \\
x^{3}: & 3\left(\frac{g_{1}}{M}+3\right) a_{3}+2 \cdot \frac{g_{2}}{M} a_{2}-3 a_{1}
\end{aligned}
$$

if $|x|<1$. Especially, the lowest power of $h(x)$ is the term $\left(\frac{g_{1}}{M}+1\right)$. Let $y_{n}(x)=\sum_{n}^{\infty} a_{k} x^{k}$ for $n \in \mathbb{N}$, i.e., $a_{k}=0$ for $k \leqq n$. Then the lowest power of $L y_{n}(x)$ is the term $\left(\frac{g_{1}}{M}+n\right) n a_{n} x^{n}$. Setting $L y_{n}(x)=\lambda_{n} y_{n}(x)$ and comparing the coefficients, we then get the eigenvalue

$$
\lambda_{n}=\left(\frac{g_{1}}{M}+n\right) n
$$

corresponding to the function $y_{n}$. In turn, once the eigenvalue is specified, the coefficients $a_{n+1}, a_{n+2}, \ldots$, are determined recursively by comparing the coefficients of
$L y_{n}$ and $\lambda_{n} y_{n}$. Although the above arguments hold true only for $|x|<1, \lambda_{n}$ is the eigenvalue corresponding to $y_{n}$ for the entire domains of definition, $x \in(-\infty, 0)$ and $(0, \infty)$, respectively. Thus the smallest eigenvalue of $L$ is $\lambda_{1}=\frac{g_{1}}{M}+1$. Now, $L$ can be expressed as $L y=-\frac{1}{r}\left(p y^{\prime}\right)^{\prime}$ with $p(x)=\exp \left(-\int \frac{h(x)}{x^{2}} \mathrm{~d} x\right)$, i.e.,

$$
p(x)=\frac{1+x^{2}}{|x|^{\frac{g_{1}}{M}}} \mathrm{e}^{-\frac{g_{2}}{M} x},
$$

and $r(x)=\frac{p(x)}{x^{2}}$, satisfying the boundary conditions $y(0, t)=0, y(x, t) \rightarrow 0$ as $x \rightarrow \infty, \frac{\partial}{\partial x} y(x, t) \rightarrow 0$ as $x \rightarrow-\infty$, and $y(x, 0)=\frac{M}{2} f(x)$. Thus, we have a SturmLiouville problem on each interval $(-\infty, 0)$ and $(0, \infty)$ separately, possessing the eigensolutions $y_{n}$ corresponding to the eigenvalues $\lambda_{n}$ of (38), and our initial problem (28) has the solutions

$$
\begin{aligned}
T(\theta, t)= & \int_{J^{ \pm}} G(\theta, \alpha, t) r(\alpha) f(\alpha) \mathrm{d} \alpha \\
& -\int_{0}^{t} \int_{J^{ \pm}} G(\theta, \alpha, t-\tau) r(\alpha) \mathrm{d} \alpha \mathrm{d} \tau
\end{aligned}
$$

with

$$
G(\theta, \alpha, t)=\sum_{n=1}^{\infty} \frac{y_{n}(\theta) y_{n}(\alpha)}{\left\|y_{n}\right\|^{2}} \mathrm{e}^{-\left(g_{1}+M n\right) n t / 2}
$$

where $\theta=2 \arctan x$ and $\alpha=2 \arctan x^{\prime}$ according to (30), and $\left\|y_{n}\right\|^{2}=\int_{J^{ \pm}} r y_{n}^{2} \mathrm{~d} x$. Hence an arbitrarily given initial state $\theta_{0}$, i.e., $f(\theta)=\delta_{\theta_{0}}(\theta)$, is pushed exponentially fast in time $t$ to one of the final states $\theta= \pm \pi$, or $x=0$, because

$$
\begin{aligned}
T(\theta, t) & =r\left(\theta_{0}\right) \sum_{n=1}^{\infty} \frac{y_{n}(\theta) y_{n}\left(\theta_{0}\right)}{\left\|y_{n}\right\|^{2}}\left(\frac{1+\lambda_{n}}{\lambda_{n}} \mathrm{e}^{-\lambda_{n} t}-\frac{1}{\lambda_{n}}\right) \\
& \leqq 2 \mathrm{e}^{-\lambda_{1} t} r\left(\theta_{0}\right) \sum_{n=1}^{\infty} \frac{y_{n}(\theta) y_{n}\left(\theta_{0}\right)}{\left\|y_{n}\right\|^{2}}
\end{aligned}
$$

i.e., Eq. (22), for the smallest eigenvalue $\lambda_{1}$ in (38).

Example 5 Consider the the case $g_{1}=M, g_{2}=-\frac{M}{2}$ given in [1, Fig. $3(\mathrm{~b})$ ]. Then the eigenvalues $\mu$ of the expected time $T$ to set the quantum system into the state $|1\rangle\langle 1|$ given by (38) are bounded by $\mu \geqq \frac{M}{2} \lambda_{1}=M$. Therefore, the greater the measurement rate $M$, the greater is the smallest possible eigenvalue of $T$.

\section{DISCUSSION}

In this article, a stability criterion for a quantum feedback control process has been introduced, as well as its expected running time in case of perfect detection efficiency. In Theorem 2, a sufficient limit for the experimental control parameters leading to globally stable quantum 
feedback control processes acting on a spin- $\frac{1}{2}$ quantum system are given. The proof consists of the application of Lyapunov's method to the stochastic differential equation governing the quantum state evolution under feedback control.

In Theorem 4 it is shown that, for perfect detection efficiency, the quantum feedback control process terminates even exponentially fast in time. The proof bases on the power series ansatz $y=\sum_{n}^{\infty} a_{k} x^{k}$ for the derived equation $\frac{\partial T}{\partial t}-\frac{M}{2} L T=-1$ determining the expected time $T$, yielding eigenfunctions $y_{n}$ with corresponding positive eigenvalues $\lambda_{n}$ by Eq. (38). Mathematically, $T$ is the expected first exit time. Theorem 4 implies that the expected running time $T_{\mathrm{qfc}}$ of a quantum feedback control process does not depend on the probability neither of the desired state, nor of the initial state. Former numerical investigations indicate that the running time of quantum feedback control algorithms, and thus $T_{\mathrm{qfc}}$, is about a tenth of the decoherence time [26] up to the order of the decoherence time [32].

Thus we are left with the unsatisfactory situation that the general case of quantum feedback control with imperfect detection efficiency could not yet be proved to be exponentially stable, in contrast to the marginal case of pure states and perfect detection efficiency. Of course, the fact that a Lyapunov function proving exponential stability could not be found does not mean that there does not exist any at all. However, by the proof of Theorem 2 such a Lyapunov function cannot be of the form (17), since with $\boldsymbol{x}=\left(\begin{array}{l}\lambda \\ \nu\end{array}\right) \in D^{2},|\boldsymbol{x}|^{2}=\lambda^{2}+\nu^{2}=2 r \nu=$ $2 r^{2}(1+\cos \theta)$, i.e., with Eq. (20),

$$
\frac{f_{\min }}{2}|\boldsymbol{x}|^{2} \leqq \mathscr{L} V(\boldsymbol{x}) \leqq \frac{f_{\max }}{2}|\boldsymbol{x}|^{2} \leqq 0,
$$

but $V$ has a linear term such that there does not exist a constant $\alpha>0$ satisfying $\mathscr{L} V(\boldsymbol{x}) \leqq-\alpha V(\boldsymbol{x})$. By Theorem 4, however, a Lyapunov function may exist satisfying this criterion at least for pure states. Thus for future mathematical investigation the question remains: Can quantum feedback control, including purification of a mixed state, be exponentially stable in general?
[1] R. van Handel, J. K. Stockton, and H. Mabuchi, IEEE Trans. Automat. Control 50, 768 (2005), quant-ph/0402136

[2] A. Goswami, Quantum Mechanics (Wm. C. Brown publishers, Dubuque, IA, 1997), 2nd ed.

[3] M. A. Nielsen and I. L. Chuang, Quantum Computation and Quantum Information (Cambridge University Press, Cambridge, 2000).

[4] M. Dušek and V. Bužek, Phys. Rev. A 66, 022112 (2002).

[5] V. P. Belavkin, J. Multivariate Anal. 42, 171 (1992).

[6] V. P. Belavkin, Comm. Math. Phys. 146, 611 (1992).

[7] V. P. Belavkin, Theory Probab. Appl. 38, 573 (1994).

[8] H. M. Wiseman, Phys. Rev. A 49, 2133 (1994).

[9] M. A. Armen, J. K. Au, J. K. Stockton, A. C. Doherty, and H. Mabuchi, Phys. Rev. Lett. 89, 133602 (2002), quant-ph/0204005

[10] J. K. Stockton, M. Armen, and H. Mabuchi, J. Opt. Soc. Am. B 19, 3019 (2002).

[11] J. K. Stockton, R. van Handel, and H. Mabuchi, Phys. Rev A70, 022106 (2004), quant-ph/0402137

[12] H. M. Wiseman, S. Mancini, and J. Wang, Phys. Rev. A 66, 013807 (2002), quant-ph/0201145.

[13] H. M. Wiseman and J. F. Ralph, New Journal of Physics 8, 90 (2006), quant-ph/0603062.

[14] H.-S. Goan and G. J. Milburn, Phys. Rev. B 64, 235307 (2001), cond-mat/0103005.

[15] T. Fujisawa, T. Hayashi, and Y. Hirayama, J. Vac. Sci. Technol. B 22, 2035 (2004).

[16] A. Shnirman and G. Schön, Phys. Rev. B 57, 15400 (1998).

[17] S. A. Gurvitz, Phys. Lett. A 311, 292 (2003), quant-ph/0303177

[18] S. A. Gurvitz and G. P. Berman, Phys. Rev. B 72, 073303 (2005), cond-mat/0408051.

[19] B. Sherman and G. Kurizki, Phys. Rev. A 45, R7674 (1992).
[20] G. Harel, G. Kurizki, J. K. McIver, and E. Coutsias, Phys. Rev. A 53, 4534 (1996).

[21] M. Fortunato, W. P. Schleich, and G. Kurizki, acta physica slovaca 46, 381 (1996).

[22] M. Fortunato, G. Kurizki, and W. P. Schleich, Phys. Rev. A 59, 714 (1999), quant-ph/9808049.

[23] D. Oblak, P. G. Petrov, C. L. Garrido, W. Tittel, A. K. Vershovski, J. K. Mikkelsen, J. L. Sørensen, and E. S. Polzik, Phys. Rev. A 71, 043807 (2005), quant-ph/0312165.

[24] E. Joos, H. D. Zeh, C. Kiefer, D. Giulini, J. Kupsch, and I.-O. Stamatescu, Decoherence and the Appearance of a Classical World in Quantum Theory (Springer-Verlag, Berlin Heidelberg, 2003), 2nd ed.

[25] L. K. Thomsen, S. Mancini, and H. M. Wiseman, J. Phys. B: At. Mol. Opt. Phys. 35, 4937 (2002), quant-ph/0208160.

[26] C. Ahn, A. C. Doherty, and A. J. Landahl, Phys. Rev. A 65, 042301 (2002), quant-ph/0110111.

[27] B. Øksendal, Stochastic Differential Equations. An Introduction with Applications (Springer-Verlag, Berlin Heidelberg New York, 1998), 5th ed.

[28] R. Z. Has'minskī̄, Stochastic Stability of Differential Equations (Sijthoff \& Noordhoff, Alphen Rockville, 1980).

[29] D. J. Higham, X. Mao, and A. M. Stuart, LMS J. Comput. Math 6, 297 (2003), http://www.lms.ac.uk/jcm/6/lms2003-014/

[30] C. W. Gardiner, Handbook of Stochastic Methods for Physics, Chemistry and the Natural Sciences (SpringerVerlag, Berlin Heidelberg, 1990), 2nd ed.

[31] P. Wilmott, Derivatives. The Theory and Practice of Financial Engineering (John Wiley, Chichester, 1998).

[32] D. J. Atkins, Z. Brady, K. Jacobs, and H. M. Wiseman, Europhys. Lett. 69, 163 (2005), quant-ph/0408080. 